$08,10,11$

\title{
Роль гетерогенной нуклеации в активации клеток крови
}

\author{
() И.В. Приходько, Г.Т. Гурия \\ Национальный медицинский исследовательский центр гематологии \\ Министерства здравоохранения Российской Федерации, \\ Москва, Россия \\ "E-mail: guria@blood.ru
}

Поступила в Редакцию 16 июля 2019 г.

В окончательной редакции 16 июля 2019 г.

Принята к публикации 25 июля 2019 г.

\begin{abstract}
Рассматриваются процессы кластеризации рецепторов на поверхности клеток крови в рамках теории нуклеации. При отсутствии лигандов характерное время гомогенной нуклеации при кластеризации рецепторов полагалось много большим времени жизни клетки. Варьируя константы кинетики образования и разрыва связей при кластеризации рецепторов, удалось описать условия активации, экспериментально наблюдаемые для нескольких видов клеток крови. Показано, что характерное время гетерогенной нуклеации, ведущей к кластеризации рецепторов в присутствии экзогенных лигандов, может быть менее одной минуты. Построены параметрические диаграммы, отображающие воздействие ряда рассмотренных лигандов на рецепторную систему. Обсуждается возможное клиническое значение полученных результатов.
\end{abstract}

Ключевые слова: рецепторы, распознавание, нуклеация, фазовый переход, критический кластер.

DOI: $10.21883 /$ FTT.2020.01.48725.46ks

\section{1. Введение}

Все типы клеток крови образуются в результате последовательных актов дифференцировки и пролиферации стволовых клеток [1]. Переходы от одного типа дифференциации к другому происходят в результате активации клеток и проявляются в резком изменении характера их поведения [2]. При этом для ряда клеток, в том числе лимфоцитов и тромбоцитов, показано, что в процессе активации существенную роль играют рецепторы, расположенные на поверхности клеток, которые пространственно сближаются, формируя кластеры [3-6]. У $T$-лимфоцитов формирование кластеров мембранных рецепторов инициирует запуск внутриклеточных автокаталитических процессов. При этом мембранные кластеры, по сути, выступают в роли затравочных каталитических подложек [7].

В настоящей работе процесс кластеризации рецепторов рассматривается с позиций теории нуклеации [8]. В центре рассмотрения находится вопрос о том, какие типы лигандов способны пороговым образом инициировать кластеризацию рецепторов. В ходе рассмотрения ставилась цель оценить величину вероятности формирования критического нуклеационного зародыша на поверхности клеток крови. При этом в рамках развиваемого подхода кластеризация рецепторов, сопровождающаяся активацией клеток, трактуется как одна из форм гетерогенной нуклеации $[9,10]$.

Такой подход позволил спроецировать ряд понятий и подходов, полученных ранее в рамках общей теории гетерогенной нуклеации [10-12] на процессы формирования закритических мембранных кластеров. В частности, удалось получить аппроксимационное выражение для линий уровня потока закритических кластеров рецепто- ров в зависимости от параметров взаимодействия рецептора и лиганда (см. выражение (1) разд. Результаты). С использованием этого выражения удалось объяснить механизм, посредством которого натуральные киллеры (вид клеток крови) способны распознавать ситуации с резко пониженным содержанием соответствующих им функциональных лигандов [13].

Построена параметрическая диаграмма для мультимерных лигандов, которая позволяет интерпретировать данные об активации тромбоцитов фактором фон Виллебранда [14]. Кроме того, в работе построена параметрическая диаграмма для случая побочных лигандов, отличающихся от основного константой диссоциации. В рамках предложенного подхода обсуждаются возможности трактовки ранних стадий иммунного ответа. В частности, были рассмотрены вопросы о чувствительности и специфичности $T$-лимфоцитов [15].

\section{2. Кинетическая модель}

Как известно, понятие о критическом зародыше лежит в основе теории фазовых переходов первого рода [16]. Вероятность флуктуационного образования нуклеационного зародыша во многих системах, собственно, и определяет время жизни метастабильной фазы $[9,11,17]$. Образование макромолекулярных кластеров на существенно двумерных объектах, в том числе на биологических мембранах, в современной биофизике рассматривается как фазовый переход первого рода [18].

В настоящей работе мы будем исходить из того, что само по себе формирование любого кластера рецепторов является стохастическим процессом. В теории фазовых переходов первого рода принято говорить о 


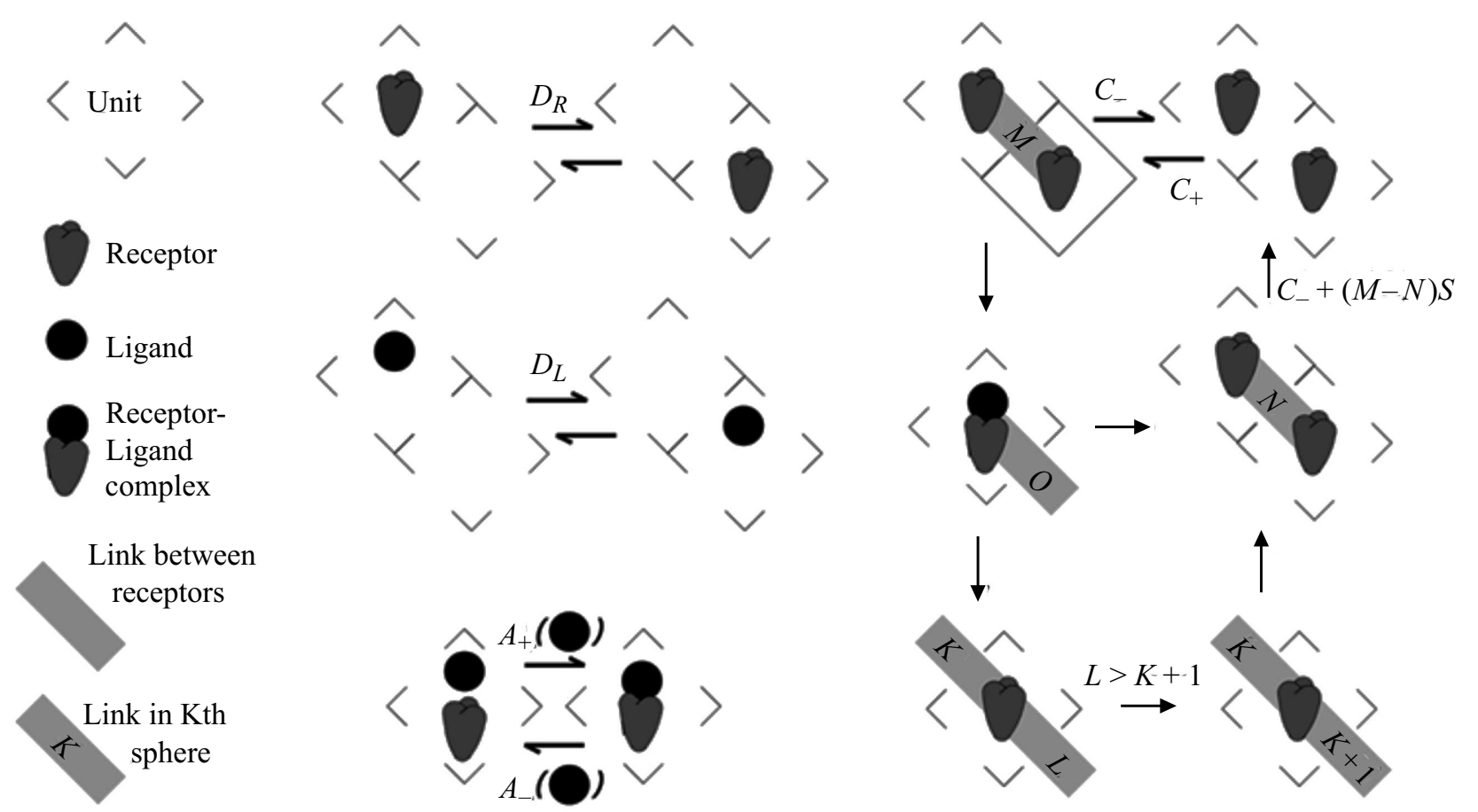

Рис. 1. Правила перехода в клеточном автомате, реализующем кластеризацию рецепторов.

вероятности формирования критических нуклеационных затравок [10]. В гомогенных системах в качестве такого рода затравок могут выступать локальные флуктуации плотности [11]. В гетерогенных системах роль затравок могут играть разнообразные „примеси“ [9].

Имея это в виду, полагалось, что функциональные лиганды, при определенных условиях, способны выступать в качестве примесных затравок, вызывающих кластеризацию рецепторов. По развиваемым представлениям, необходимым условием для формирования критического кластера является контакт одного из входящих в кластер рецепторов с лигандом. Последний при этом выступает в качестве гетерогенного („примесного“) центра нуклеации. Присоединение к данному центру других рецепторов полагалось статистически предпочтительным за счет локального увеличения времени жизни связей в кластере в некоторой области вокруг рецептора, ассоциированного с лигандом. Конкретно предполагалось, что энергия диссоциации отдельных связей между рецепторами в кластере уменьшается по мере удаления от нуклеационного центра [19].

Для несимметричных (не цилиндрических по форме) кластеров, состоящих из реальных рецепторов, в качестве индикатора нуклеации использовалось общее количество связей между входящими в кластер рецепторами. До образования критического зародыша, распределение отдельных рецепторных кластеров по количеству связей в них предполагалось близким к термодинамическому равновесию, а сумма размеров всех кластеров полагалась неизменной во времени. Появление хотя бы одного закритического зародыша влекло за собой рост количества связей [20]. Такой подход позволил определять время нуклеации на основании кинетических кривых, отображающих изменение количества связей во времени.

В настоящей работе для моделирования пространственных эффектов была построена модель типа „ячеистый автомат“ [21]. Имея в виду сопоставимость размера рецепторов и лигандов, поверхность клетки разбивалась на ячейки такого размера, что в каждой из них могло находится не более одной лиганд-рецепторной пары.

Вероятность перехода свободных рецепторов между ячейками размера $r$ за временной шаг $\tau$ задавалась в виде $P_{d} \propto \exp \left(-r^{2} / D_{R} \tau\right)$, где в качестве параметра $D_{R}$ выступал эффективный коэффициент диффузии. Рецепторы, ассоциированные с лигандом или связанные с другими рецепторами, полагались неподвижными. Аналогично для лигандов вводился коэффициент латеральной диффузии $D_{L}$. Общее количество рецепторов полагалось равным $N_{R}$, а лигандов $-N_{L}$. Кинетический расчет стартовал со случайного начального распределения рецепторов и лигандов по ячейкам.

Каждой паре рецептор-лиганд ставились в соответствие энергии ассоциации $A_{+} k_{\mathrm{B}} T$ и диссоциации $A_{-} k_{\mathrm{B}} T$, где $k_{\mathrm{B}}-$ постоянная Больцмана, а $T-$ абсолютная температура. Вероятность ассоциации за единицу времени в ячейке, содержащей одновременно и рецептор, и лиганд, задавалась выражением $a_{A+} \propto \exp \left(-A_{+}\right)$. А диссоциации соответственно $-a_{A-} \propto \exp \left(-A_{-}\right)$.

Энергия образования связи между рецепторами в соседних ячейках $-C_{+} k_{\mathrm{B}} T$ полагалась постоянной. Вероятность в единицу времени образования связи между такими рецепторами задавалась выражением $a_{C+} \propto \exp \left(-C_{+}\right)$. В отсутствии лиганда энергия разрыва 


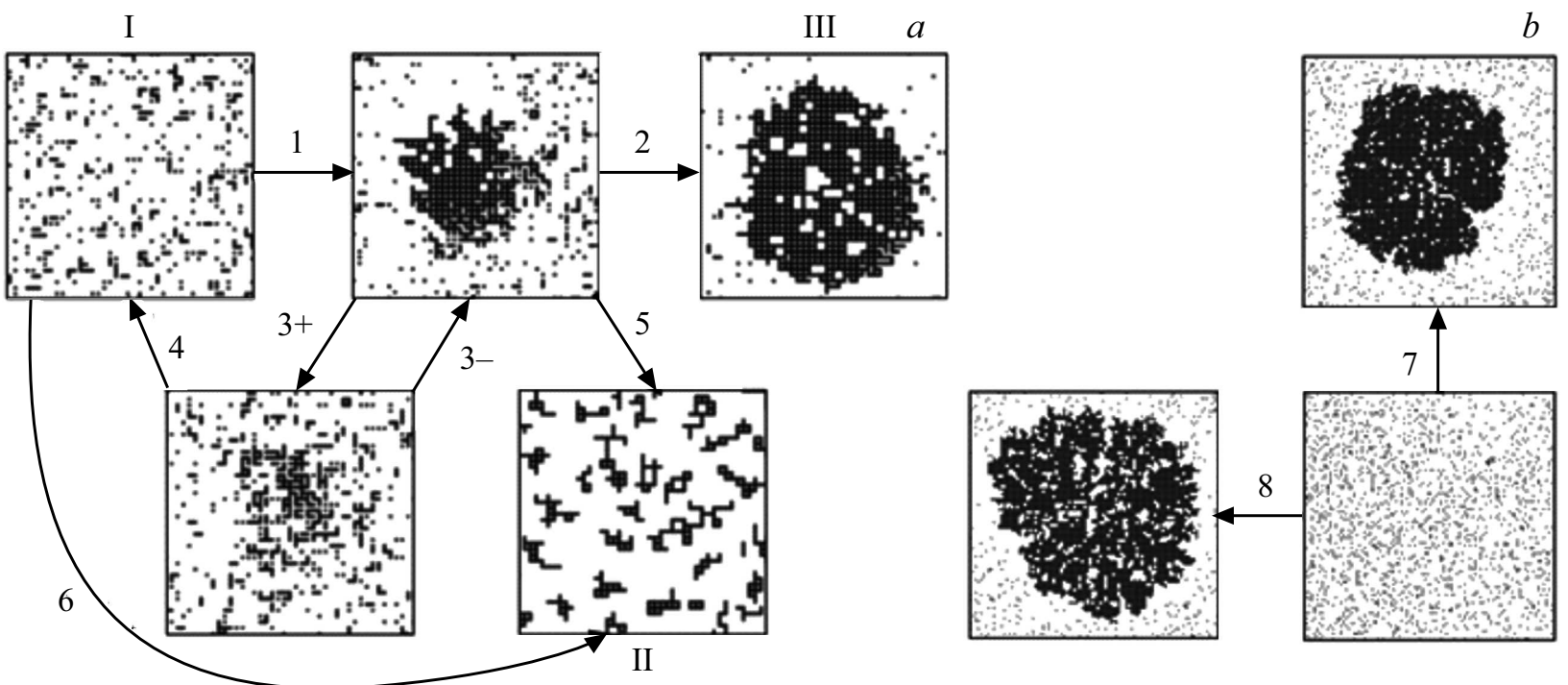

Рис. 2. $a$ - возможные переходы между различными состояниями системы рецепторов. Переход 1 - образование докритического кластера, 2 - образование гигантского кластера, 3 - обратимый коллапс связности кластера, 4 - полное разрушение докритического кластера, 5,6 - падение перенасыщенности до образования гигантских кластеров. $b-$ кластеры с разной скоростью роста, а значит различной термализованностью формы. Переход 7 - образование термализованного кластера, 8 образование „игольчатого“ кластера.

связи между рецепторами полагалась равной $C_{-} k_{\mathrm{B}} T$. Соответственно вероятность в единицу времени разрыва связи между рецепторами в соседних ячейках вычислялась по формуле $a_{C-} \propto \exp \left(-C_{-}\right)$.

Мы предполагали, что рецептор, ассоциируясь с лигандом, конформационно изменяется, за счет чего повышается энергия разрыва связи между ним и ближайшими из соседних рецепторов на величину $M S k_{\mathrm{B}} T$, где $M$ - целое число. Кроме того, энергия разрыва связей между рецепторами, расположенными в последовательных „координационных сферах“, полагалась линейно убывающей в зависимости от расстояния до центра. В $K$-й по счету сфере эта энергия полагалась равной $(M-K) S k_{\mathrm{B}} T$, где $0 \leq K<M$. Вероятность в единицу времени разрыва связей рецепторов, находящихся в $K$-й координационной сфере от ближайшего ассоциированного рецептора, задавалась выражением $a_{C K-} \propto \exp \left(-C_{-}-(M-K) S\right)$.

Вышеприведенные правила исчерпывающим образом задают как перечень возможных состояний в рассматриваемой рецепторной системе, так и набор „правил перехода“ между ними. С точки зрения теории игр, мы имеем дело с полностью определенной конечномерной динамической системой [22,23]. Вводимые в настоящей работе понятия о структуре объектов при кластеризации рецепторов и правила перехода в схематичной форме представлены на рис. 1.

\section{3. Нуклеация}

В результате проведения расчетов выяснилось, что для рецепторной системы характерны три состояния:
I - „газообразное“ состояние из уединенных рецепторов, II - стабильное состояние большого числа маленьких кластеров и III - метастабильное „газообразное“ состояние, приводящее к образованию „гигантских“ кластеров (рис. 2,a). При этом „гигантскими“ кластерами мы называли те, в которых сосредоточено количество рецепторов соизмеримое с их общим числом в системе $N_{R}$. В такого рода случаях некоторая область поверхности клетки имеет плотность рецепторов кратно превосходящую начальную. Полагалось, что данная область способна выступать в качестве затравочной каталитической подложки для процессов внутриклеточной активации [7].

В зависимости от параметров кластеризации $\left(N_{R}, C_{+}, C_{-}\right)$рецепторы, не имеющие контактов с лигандами, могут образовывать как цилиндрические, так и более причудливые „игольчатые“ и „узорчатые“ кластеры (рис. 2,b). Наибольший интерес для распознавания лиганда представляют те значения параметров $\left(N_{R}, C_{+}, C_{-}\right)$, когда система находится в метастабильном состоянии.

Отвечающие формированию гигантских кластеров параметры взаимодействия лигандов и рецепторов $\left(N_{L}, A_{+}, A_{-}\right)$, показаны на рис. 3. На данном рисунке легко видеть три основных типа зависимостей потока закритических кластеров от количества лигандов $N_{L}$ при разных значениях параметра $A_{-}$и $A_{+}$.

Видно, что при значениях параметров $\left(A_{+}, A_{-}\right)$, отвечающих случаю $(a)$, кластеризация имеет место в строго ограниченных как сверху, так и снизу интервалах изменения величины $N_{L}$ (сплошной график рис. 3 ). Для значений параметров взаимодействия лиганда и рецептора $\left(A_{+}, A_{-}\right)$, отвечающих случаю $(b)$, кластери- 


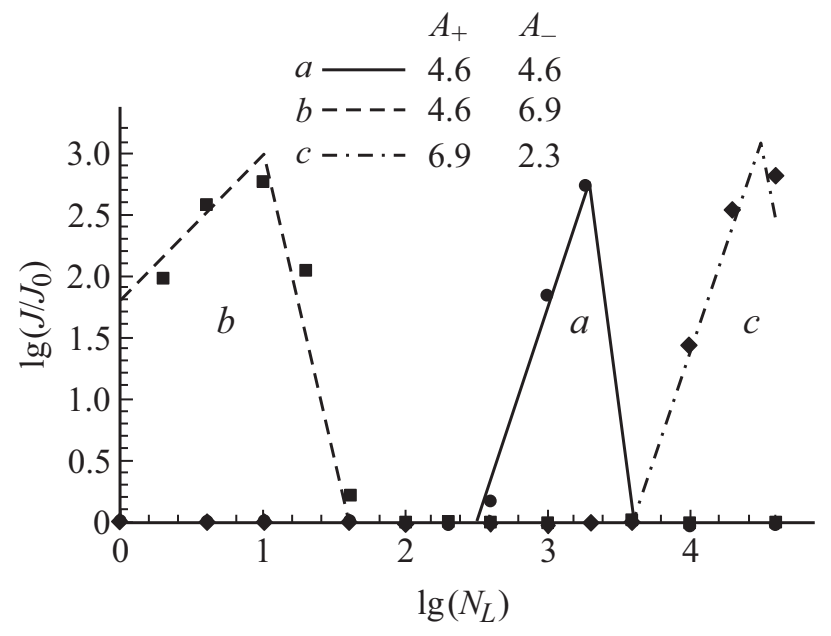

Рис. 3. Зависимость логарифма потока образования закритических кластеров рецепторов $\lg \left(J / J_{0}\right)$ от логарифма количества лиганда с разными константами диссоциации и ассоциации лиганда и рецептора $\lg \left(N_{L}\right)$.

зация возможна при сколь угодно малой концентрации лигандов (рис. 3, штриховой график). По всей видимости возможности натуральных киллеров „чувствовать“ отсутствие лигандов удается рационально объяснить тем, что параметры взаимодействия их рецепторов и лигандов отвечают случаю $(b)$ [13].

\section{4. Параметрические диаграммы}

Как видно из зависимостей, представленных на рис. 3, образование гигантских рецепторных кластеров может иметь место, если количество лигандов попадает в достаточно узкий интервал значений. Представленная на рис. 4, $a$ параметрическая диаграмма отвечают случаю $(a)$. Видно, что увеличение числа рецепторов $\left(N_{R}\right)$ делает возможным снижение необходимого для „гигантской“ кластеризации критического количества лигандов $\left(N_{L}\right)$. Это значит, что клетки крови в принципе могут изменять свою чувствительность существенным образом, как за счет регулирования экспрессии рецепторов, так и их деградации.

Лиганды, вызывающие активацию у тромбоцитов и $B$-лимфоцитов, во многих случаях имеют поли- и мультимерные структуры [24]. Так тромбоциты способны реагировать на размотанные в достаточной степени по их поверхности макромолекулы факторов фон Виллебранда $[14,25]$. На рис. 4, $b$ построена параметрическая диаграмма для мультмерного лиганда при значениях параметра $A_{+}=4.7$. Легко видеть, что при высокой мультимерности (больше 30 мономеров)
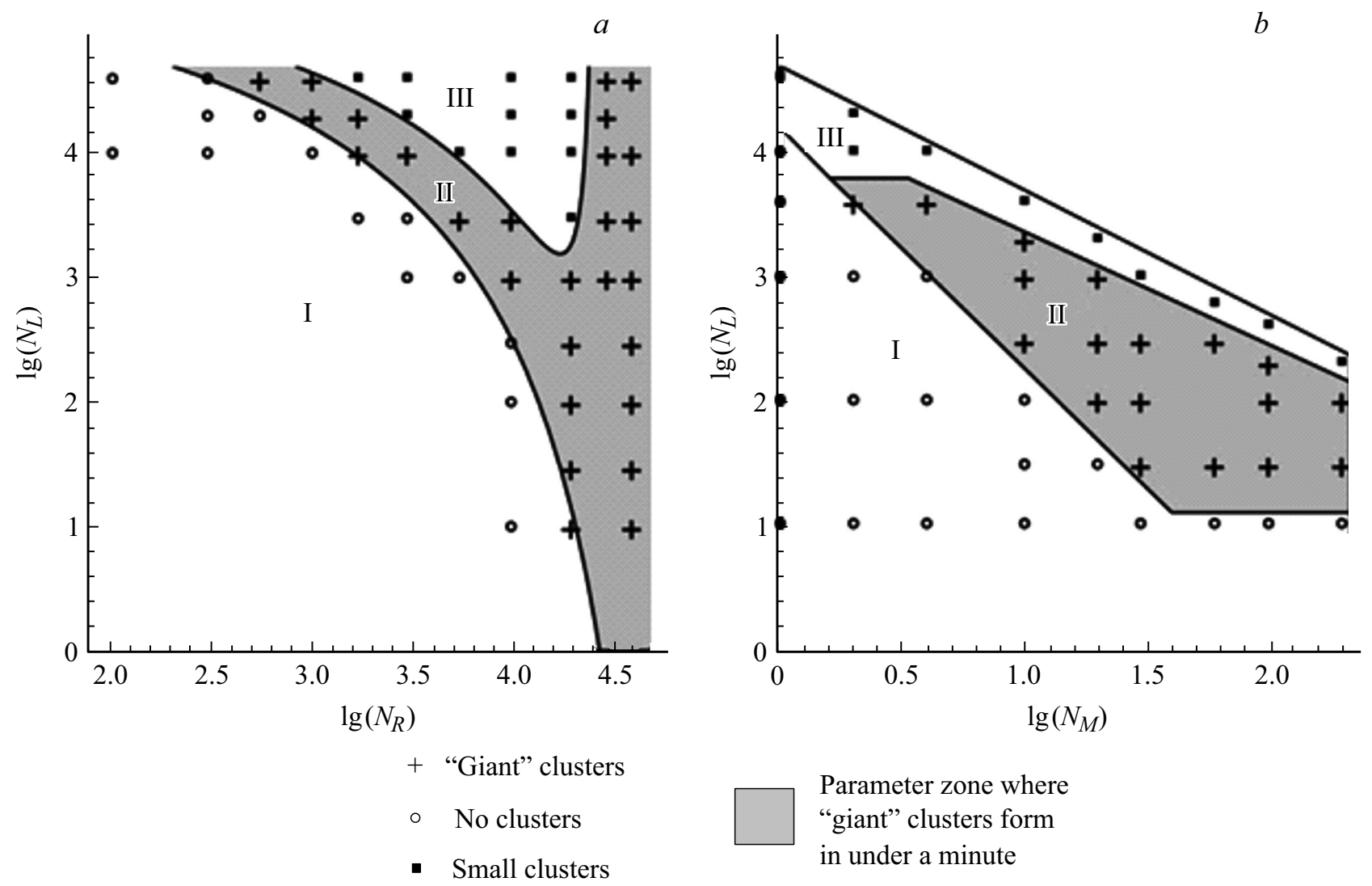

Рис. 4. Параметрические диаграммы состояния системы рецепторов в осях логарифмов количества рецепторов и лигандов. Закрашенная область отображает параметры, при которых в рассмотренной модели наблюдаются активация. 
$a$

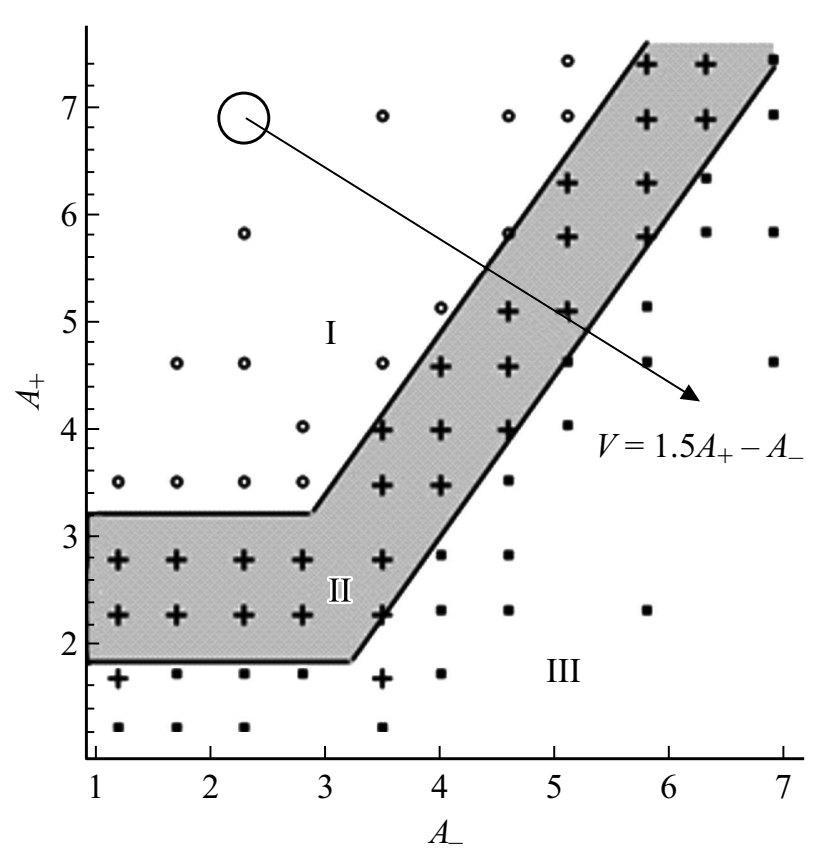

+ "Giant" clusters

- No clusters

- Small clusters Secondary ligand $b$

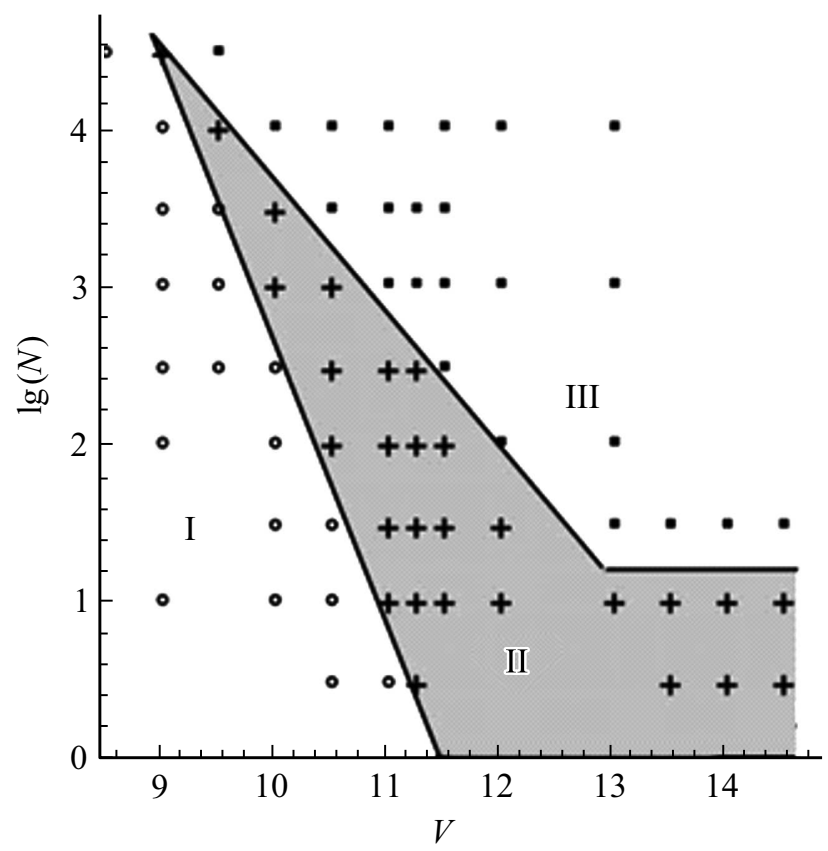

Parameter zone where "giant" clusters form in under a minute

Рис. 5. Параметрические диаграммы состояний системы рецепторов в присутствии побочного лиганда. $a-$ в осях параметров взаимодействия рецептора и лиганда и введение параметра $V=1.5 A_{+}-A_{-}$, описывающего состояние системы в широких пределах. Кругом отмечены параметры побочного лиганда. $b-$ в осях вновь введенного параметра $V$ и количества лиганда.

необходимое для активации количество лигандов практически не зависит от степени мультимерности. При снижении мультимерности необходимое для активации количество лигандов резко растет.

Параметры модели, которые использовались в расчетах

\begin{tabular}{c|c|l}
\hline Параметр & Значение & \multicolumn{1}{|c}{ Пояснение } \\
\hline$D$ & $0.5 \mu \mathrm{m} / \mathrm{s}$ & Коэффициент диффузии \\
$N_{R}$ & 10000 & Количество рецепторов \\
$C_{+}$ & 4.6 & Б/p энергия связывания \\
$C_{-}$ & 2.3 & Б/p энергия разрыва связи \\
$S$ & 0.36 & Б/p энергия стабилизации связи \\
$M$ & 20 & Количество координационных сфер \\
& & в которых происходит стабилизация \\
$A_{+}$ & 4.6 & Б/р энергия ассоциации рецептора \\
& 4.6 & и лиганда \\
$A_{-}$ & 1 & Б/р энергия диссоциации рецептора \\
$N_{M}$ & $1 \mu \mathrm{m}$ & Муганда \\
$\tau_{*}$ & $4 \mu$ & Размер расчетной области \\
$r$ & $1 \mu$ & Размер ячейки автомата \\
$\tau$ & Шаг автомата
\end{tabular}

Примечание. Б/p - безразмерная.
У многих рецепторов имеются побочные лиганды, т.е. вещества способные связываться с рецепторами с меньшей константой ассоциации, чем у главного лиганда. Особенно это заметно у рецепторов $T$-лимфоцитов, которые способны различать маленькие фрагменты из 7-11 аминокислот, прикрепленные к их лигандам. Проведенный анализ показал, что добавление побочного лиганда (в дополнение к главному), практически не влияет на форму параметрической диаграммы для главного лиганда, в тех случаях, когда параметры находятся в области I и расположены далеко от ее границы (рис. 5, $a$ ).

Построив параметрическую диаграмму, приведенную на рис. 5, $a$, мы обнаружили, что за исключением области с чрезвычайно малой энергией образования связи, линии уровня потока закритических кластеров представляют из себя прямые линии с коэффициентом наклона 1.5. С учетом этого, процессы перехода к кластеризации рецепторов имеет смысл описывать одним „параметром порядка“ $-V$, задаваемым уравнением (1)

$$
V=1.5 A_{+}-A_{-} .
$$

На рис. 5, $b$ представлена параметрическая диаграмма, которая показывает отклик системы на изменение 
количества и характеристики главного лиганда. Видно, что изменение индекса $V$ в 3 раза делает возможным регистрацию всего одной молекулы лиганда. Все значения параметров, использованных в расчетах, не указанные явно в основном тексте статьи, приведены в таблице.

\section{5. Дискуссия}

Проанализирован механизм кластеризации рецепторов на поверхности нескольких типов клеток крови. Показано, что в условиях физиологической нормы быстрота кластеризации рецепторов имеет решающее значение для эффективного функционирования клеток и определяет их способность к быстрому реагированию на внешние сигналы. Следует отметить, что для ряда следящих биологических систем было установлено, что их основное состояние является метастабильным, что обеспечивает передачу сигналов в нейронах [26] и в плазменном звене системы свертывания крови [27].

Клетки крови в течение своего жизненного цикла на порядки меняют свою чувствительность, иногда обратимо [28]. Обычно этот феномен связывают с механизмами, отвечающими за изменение клетками общего количества своих рецепторов. В свете полученных результатов представляется важным отметить, что чувствительность существенным образом может изменяться и в зависимости от степени ,рыхлости“ кластеров (рис. 2,b). Представляется, что мембранные системы даже с небольшим числом рецепторов за счет процессов стремительной кластеризации последних способны разворачивать высокочувствительные кластерные „антенны“ для первичного приема сигналов.

Посредниками для связи между рецепторами могут выступать как мембранные рафты, так и элементы актинового цитоскелета. В частности, гидрофобные примеси могут значительно увеличивать стабильность рафтов [18]. При этом рецепторы могут конформационно изменяться в ходе кластеризации в своей структуре, „разворачивая“ гидрофобные участки в сторону мембраны. В области межклеточных контактов наблюдается активное перестроение цитоскелета. В такого рода случаях ассоциированные с лигандами рецепторы замедляют движение цитоскелета относительно мембраны.

К числу клинически важных следствий построенной модели относится заключение о том, что для перевода клетки из метастабильного состояния III типа в состояние II типа достаточно более 30 лигандов с индексом $\geq 7$. Представляется, что введение такого рода лигандов способно быстро купировать активационные процессы в соответствующих клетках. К числу таких ситуаций, повидимому, относятся остановка стремительного тромбообразования, коррекция острых гипераллергических реакций, а также быстрые реакции „свой-чужой“ в трансплантатологии.

\section{6. Заключение}

Построена модель кластеризации рецепторов на поверхности клеток крови. Результаты расчетов обсуждаются в свете подходов, разработанных в рамках общей теории гетерогенной нуклеации. Построен ряд параметрических диаграмм, позволяющих объяснить характер реакции нескольких типов клеток крови на экзогенные стимулы.

Выдвинуто предположение о том, что рецепторные системы ряда клеток крови (в том числе тромбоцитов, лимфоцитов и лейкоцитов) в пределах физиологической нормы являются метастабильными. Как следствие, акцептирование клетками экзогенных стимулов может осуществляться по механизму гетерогенной нуклеации, имея своим итогом образование „гигантских“ кластеров. Последние при этом выступают в качестве затравочных каталитических подложек для оперативной трансляции сигналов внутрь клеток и дальнейших внутриклеточных этапов активации.

\section{Финансирование работы}

Работа была частично поддержана Российским научным фондом (грант № 19-11-00260).

\section{Конфликт интересов}

Авторы заявляют, что у них нет конфликта интересов.

\section{Список литературы}

[1] J.E. Till, E.A. McCulloch. Rad. Res. 14, 213 (1961).

[2] Р. Шмидт, Г. Тевс. Физиология человека. Мир, М. (2005). T. $2.314 \mathrm{c}$.

[3] W.M. Isenberg, R.P. McEver, D.R. Phillips, M.A. Shuman, D.F. Bainton. J. Cell Biology 104, 1655 (1987)

[4] B. Wójciak-Stothard, L. Williams, A.J. Ridley. J. Cell Biology 145, 1293 (1999).

[5] I.I. Singer, S. Scott, D.W. Kawka, J. Chin, B.L. Daugherty, J.A. DeMartino, J. DiSalvo, S.L. Gould, J.E. Lineberger, L. Malkowitz, M.D. Miller, L. Mitnaul, S.J. Siciliano, M.J. Staruch, H.R. Williams, H.J. Zweerink, M.S. Springer. J. Virology 75, 3779 (2001).

[6] M. Eriksson, G. Leitz, E. Fällman, O. Axner, J.C. Ryan, M.C. Nakamura, C.L. Sentman. J. Exp. Medicine 190, 1005 (1999).

[7] S.V. Pageon, T. Tabarin, Y. Yamamoto, Y. Ma, P.R. Nicovich, J.S. Bridgeman, A. Cohnen, C. Benzing, Y. Gao, M.D. Crowther, K. Tungatt, G. Dolton, A.K. Sewell, D.A. Price, O. Acuto, R.G. Parton, J.J. Gooding, J. Rossy, J. Rossjohn, K. Gaus. Proc. Nat. Acad. Sci. 113, E5454 (2016).

[8] R. Becker, W. Döring. Annal. Phys. 24, 719 (1935).

[9] D. Turnbull. J. Chem. Phys. 18, 198 (1950).

[10] J.W. Schmelzer, V.M. Fokin, A.S. Abyzov, E.D. Zanotto, I. Gutzow. Int. J. Appl. Glass Sci. 1, 16 (2010).

[11] N. H. J. Fletcher. J. Chem. Phys. 29, 572 (1958).

[12] R.P. Sear. J. Phys.: Condensed Matter 19, 033101 (2007).

[13] P. Stern, M. Gidlund, A. Örn, H. Wigzell. Nature 285, 341 (1980). 
[14] M. Stockschlaeder, R. Schneppenheim, U. Budde. Blood Coagulation Fibrinolysis 25, 206 (2014).

[15] O. Feinerman, R.N. Germain, G. Altan-Bonnet. Mol. Immunology 45, 619 (2008).

[16] Я.Г. Синай. Теория фазовых переходов. Наука, М. (2006). $208 \mathrm{c}$.

[17] D. Gunton. Phase Transitions Critical Phenomena 8, 267 (1983).

[18] V.A.J. Frolov, Y.A. Chizmadzev, F.S. Cohen, J. Zimmerberg. Biophys. J. 91, 189 (2006).

[19] D. Bray, M.D. Levin, C.J. Morton-Firth. Nature 393, 85 (1998).

[20] T. Vicsek. Fractal growth phenomena. World scientific (1992). $510 \mathrm{p}$.

[21] M. Markus, B. Hess. Nature 347, 56 (1990).

[22] M. Eigen, R. Winkler. Laws of the game: how the principles of nature govern chance. Princeton University Press (1993). $368 \mathrm{p}$.

[23] J. Conway. Sci. Am. 223, 4 (1970).

[24] B.S. Coller, E.I. Peerschke, L.E. Scudder, C.A. Sullivan. Blood 61, 99 (1983).

[25] K.E. Zlobina, G.Th. Guria. Sci. Rep. 6, 30508 (2016).

[26] R. Fitzhugh. J. General Physiology 43, 867 (1960).

[27] Г.Т. Гурия. Наука (Изд. дом „Коммерсанть“), М. (2011). T. 9. C. 54.

[28] M. Hansson R. Kiessling, B. Andersson, K. Kärre, J. Roder. Nature 278, 174 (1979).

Редактор Д.В. Жуманов 